\title{
Dynamics of POD Modes in Wall Bounded Turbulent Flow
}

\author{
Giancarlo Alfonsi $i^{1}$ and Leonardo Primavera ${ }^{2}$ \\ ${ }^{1}$ Dipartimento di Difesa del Suolo, Università della Calabria \\ Via P. Bucci 42b, 87036 Rende (Cosenza), Italy \\ alfonsi@dds.unical.it \\ ${ }^{2}$ Dipartimento di Fisica, Università della Calabria \\ Via P. Bucci 33b, 87036 Rende (Cosenza), Italy \\ lprimavera@fis.unical.it
}

\begin{abstract}
The dynamic properties of POD modes of the fluctuating velocity field developing in the wall region of turbulent channel flow are investigated. The flow of viscous incompressible fluid in a channel is simulated numerically by means of a parallel computational code based on a mixed spectral-finite difference algorithm for the numerical integration of the Navier-Stokes equations. The DNS approach (Direct Numerical Simulation of turbulence) is followed in the calculations, performed at friction Reynolds number $\operatorname{Re}_{\tau}=180$. A database representing the turbulent statistically steady state of the flow through 10 viscous time units is assembled and the Proper Orthogonal Decomposition technique (POD) is applied to the fluctuating portion of the velocity field. The dynamic properties of the most energetic POD modes are investigated showing a clear interaction between streamwise-independent modes and quasi-streamwise modes in the temporal development of the turbulent flow field.
\end{abstract}

\section{Introduction}

The hypothesis incorporated in all turbulence theories that have been formulated in the last decades is that of the local isotropy of the small turbulent scales (Kolmogorov [1]), i.e. the postulate that the small-scale structures of turbulent flows possess universal statistical properties independent of the large scales. Local isotropy has also been enforced in most of the existing SGS (subgrid-scale) closures within the LES (Large Eddy Simulation) approach to turbulence modelling.

The verification of the hypotheses that provide the basis for turbulence theories is an issue of remarkable relevance for both theoreticians and modellers. Several reseachers provided evidence of the " $-5 / 3$ " velocity spectrum in the inertial range, while the issue of the universal statistical properties of small turbulent scales in both inertial and dissipation ranges is still controversial. One of the difficulties encountered is that of reaching values of the Reynolds number able to assure the development of a sufficiently broad spectrum of scales for the possible establishment of local isotropy of the small scales.

The properties of the velocity field has been studied numerically in homogeneous shear flow with constant mean shear by Pumir \& Shraiman [2]. They showed that for 
values of the Taylor microscale Reynolds number of order 100 the value of the derivative skewness of the velocity fluctuation in the direction of the mean flow along the direction of the mean velocity gradient, is of order 1. Garg \& Warhaft [3] studied experimentally the properties of the small-scale velocity field in homogeneous shear flow with constant mean shear. They found that there is a significant skewness (of order 1) of the derivative of the longitudinal velocity fluctuation in the direction of the mean gradient. Third-order transverse structure functions of the longitudinal velocity were found to have a scaling range, showing the existence of anisotropy at both inertial and dissipation scales. Shen \& Warhaft [4] continued the experiments. They showed that the fifth moment of the derivative of the longitudinal fluctuation in the direction of the mean gradient, is of order 10 with no diminution with the Reynolds number. Moreover, fifth- and seventh-order inertial subrange skewness structure functions are of order 10 and 100 respectively. These results show that there exists velocity anisotropy in both inertial and dissipation ranges, for the Reynolds number tested.

A more recent approach to turbulence modelling, that differs from the most used RANS (Reynolds Averaged Navier-Stokes equations) and LES (Large Eddy Simulation), involves methods for the reduction of the turbulent phenomenon to a system with a limited number of degrees of freedom. The Proper Orthogonal Decomposition (POD) is a technique that permits the extraction of appropriatelydefined modes of the flow from the background flow, that can be subsequently projected onto the system of the Navier-Stokes equations to obtain a low-order model of a given turbulent flow (Podvin \& Lumley [5], Omurtag \& Sirovich [6]). A relevant issue in this context is the investigation of the dynamic properties of the flow modes as extracted with the POD technique.

The present work addresses the issue of the dynamic characteristics of the coherent structures of turbulence in moderately turbulent channel flow, educed by applying the Proper Orthogonal Decomposition to the fluctuating portion of the velocity field. The POD modes are calculated from a numerical database assembled with the use of a parallel computational code for the numerical integration of the Navier-Stokes equations in the case of the plane channel at friction Reynolds number $R e_{\tau}=180$.

\section{Methods}

The simulations have been performed with a parallel computational code based on a mixed spectral-finite difference technique. The unsteady Navier-Stokes equations for incompressible fluids with constant properties in three dimensions and nondimensional conservative form, is considered $(i \& j=1,2,3)$ :

$$
\begin{aligned}
& \frac{\partial u_{i}}{\partial x_{i}}=0 \\
& \frac{\partial u_{i}}{\partial t}+\frac{\partial}{\partial x_{j}}\left(u_{i} u_{j}\right)=-\frac{\partial p}{\partial x_{i}}+\frac{1}{R e_{\tau}} \frac{\partial^{2} u_{i}}{\partial x_{j} \partial x_{j}}
\end{aligned}
$$

where $u_{i}(u, v, w)$ are the velocity components in the cartesian coordinate system $x_{i}(x, y, z)$. Equations (1) are nondimensionalized by the channel half-width $\delta$ for 
lenghts, wall shear velocity $u_{\tau}=\sqrt{\tau_{w} / \rho}$ for velocities, $\rho u_{\tau}^{2}$ for pressure and $\delta / u_{\tau}$ for time, being $R e_{\tau}=\left(u_{\tau} \delta / v\right)$ the friction Reynolds number.

The fields are admitted to be periodic in the streamwise $(x)$ and spanwise $(z)$ directions, and equations (1) are Fourier transformed accordingly. The nonlinear terms in the momentum equation are evaluated pseudospectrally by antitransforming the velocities back in physical space to perform the products (FFTs are used). A dealiasing procedure is applied to avoid errors in transforming the results back to Fourier space. In order to have a better spatial resolution near the walls, a grid-stretching law of hyperbolic-tangent type has been introduced for the grid points along $y$, the direction orthogonal to the walls. For the time advancement, a third-order Runge-Kutta algorithm has been implemented and the time marching procedure is accomplished with the fractional-step method. No-slip boundary conditions at the walls and cyclic conditions in the streamwise and spanwise directions have been applied to the velocity. More detailed descriptions of the numerical scheme, of its reliability and of the performance obtained on the parallel computers that have been used, can be found in Alfonsi et al. [7] and Passoni et al. [8],[9],[10].

By recalling the wall formalism, one has: $x_{i}^{+}=x_{i} u_{\tau} / \nu=x_{i} / \delta_{\tau}$, $t^{+}=t u_{\tau}^{2} / v=t u_{\tau} / \delta_{\tau}, \quad \delta^{+}=\delta / \delta_{\tau}, u^{+}=\bar{u} / u_{\tau}, R e_{\tau}=u_{\tau} \delta / v=\delta / \delta_{\tau}=\delta^{+}$, where $\bar{u}$ is streamwise velocity averaged on a $x-z$ plane and time, $\delta_{\tau}=v / u_{\tau}$ is the viscous length and $\delta / u_{\tau}$ the viscous time unit. The characteristic parameters of the numerical simulations are the following. Computing domain: $L_{x}=2 \pi \delta, L_{y}=2 \delta, L_{z}=\pi \delta$; $L_{x}^{+}=1131, L_{y}^{+}=360, L_{z}^{+}=565$. Computational grid: $N_{x}=96, N_{y}=129, N_{z}=64$. Grid spacing: $\Delta x^{+}=11.8, \Delta y_{\text {center }}^{+}=4.4, \Delta y_{\text {wall }}^{+}=0.87, \Delta z^{+}=8.8$. It can be verified that there are 6 grid points in the $y$ direction within the viscous sublayer $\left(y^{+} \leq 5\right)$. The Kolmogorov spatial microscale, estimated using the criterion of the average dissipation rate per unit mass across the width of the channel, results $\eta^{+} \approx 1.8$. After the insertion of appropriate initial conditions, the initial transient of the flow in the channel has been simulated, the turbulent statistically steady state has been reached and then calculated for a time $t=10 \delta / u_{\tau}\left(t^{+}=1800\right) .20000$ time steps have been calculated with a temporal resolution of $\Delta t=5 \times 10^{-4} \delta / u_{\tau}\left(\Delta t^{+}=0.09\right)$.

In Table 1 predicted and computed values of a number of mean-flow variables are reported $\left(U_{b}\right.$ and $U_{c}$ are the bulk mean velocity and the mean centerline velocity respectively, while $R e_{b}$ and $R e_{c}$ are the related Reynolds numbers). The predicted values of $U_{c} / U_{b}$ and $C_{f}$ are obtained from the correlations suggested by Dean [11] $\left[U_{c} / U_{b}=1.28\left(2 R e_{b}\right)^{-0.0116} ; C_{f}=0.073\left(2 R e_{b}\right)^{-0.25}\right]$ while the computed skin friction coefficient $\left[C_{f}=\left(2 \tau_{w} / \rho U_{b}^{2}\right) ; \tau_{w}=\mu(\partial U / \partial y)_{\text {wall }}\right]$ is calculated using the value of the shear stress at the wall actually obtained in the computations (a finite difference routine is used). 
Table 1. Predicted vs. computed mean-flow variables

Predicted variables

$\begin{array}{ccccccl}R e_{\tau} & R e_{b} & R e_{c} & U_{b} / u_{\tau} & U_{c} / u_{\tau} & U_{c} / U_{b} & C_{f} \\ 180 & 2800 & 3244 & 15.56 & 18.02 & 1.16 & 8.44 \times 10^{-3}\end{array}$

Computed variables

$\begin{array}{lcccccl}R e_{\tau} & R e_{b} & R e_{c} & U_{b} / u_{\tau} & U_{c} / u_{\tau} & U_{c} / U_{b} & C_{f} \\ 178.74 & 2786 & 3238 & 15.48 & 17.99 & 1.16 & 8.23 \times 10^{-3}\end{array}$

The Proper Orthogonal Decomposition is a technique that can be applied for the extraction of the coherent structures from a turbulent flow field (Berkooz et al. [12], Sirovich [13]). By considering an ensemble of temporal realizations of a velocity field $u_{i}\left(x_{j}, t\right)$ on a finite domain $D$, one wants to find which is the most similar function to the elements of the ensemble, on average. This problem corresponds to find a deterministic vector function $\varphi_{i}\left(x_{j}\right)$ such that $(i \& j=1,2,3)$ :

$$
\max _{\psi} \frac{\left\langle\left|\left(u_{i}\left(x_{j}, t\right), \psi_{i}\left(x_{j}\right)\right)\right|^{2}\right\rangle}{\left(\psi_{i}\left(x_{j}\right), \psi_{i}\left(x_{j}\right)\right)}=\frac{\left\langle\left|\left(u_{i}\left(x_{j}, t\right), \varphi_{i}\left(x_{j}\right)\right)\right|^{2}\right\rangle}{\left(\varphi_{i}\left(x_{j}\right), \varphi_{i}\left(x_{j}\right)\right)} .
$$

A necessary condition for problem (2) is that $\varphi_{i}\left(x_{j}\right)$ is an eigenfunction, solution of the eigenvalue problem and Fredholm integral equation of the first kind:

$$
\int_{D} R_{i j}\left(x_{l}, x_{l}^{\prime}\right) \varphi_{j}\left(x_{l}^{\prime}\right) d x_{l}^{\prime}=\int_{D}\left\langle u_{i}\left(x_{k}, t\right) u_{j}\left(x_{k}^{\prime}, t\right)\right\rangle \varphi_{j}\left(x_{k}^{\prime}\right) d x_{k}^{\prime}=\lambda \varphi_{i}\left(x_{k}\right)
$$

where $R_{i j}=\left\langle u_{i}\left(x_{k}, t\right) u_{j}\left(x_{k}^{\prime}, t\right)\right\rangle$ is the two-point velocity correlation tensor. To each eigenfunction $\varphi_{i}^{(n)}\left(x_{j}\right)$ is associated a real positive eingenvalue $\lambda^{(n)}$ and every member of the ensemble can be reconstructed by means of the modal decomposition $u_{i}\left(x_{j}, t\right)=\sum_{n} a_{n}(t) \varphi_{i}^{(n)}\left(x_{j}\right)$. The contribution of each mode to the kinetic energy content of the flow is given by $E=\int_{D}\left\langle u_{i}\left(x_{j}, t\right) u_{i}\left(x_{j}, t\right)\right\rangle d x_{j}=\sum_{n} \lambda^{(n)}$, being $E$ the turbulent kinetic energy in the domain $D$. In the present work the POD is used for the analysis of the fluctuating portion of the velocity field. The two homogeneous directions are handled in Fourier space so that the optimal representation of the velocity field in the statistical sense outlined above is sought in the direction normal to the solid walls. 


\section{Results}

As a result of the decomposition, $3 N_{y}(387)$ POD modes and correspondent eigenvalues have been determined for each wavenumber index pair $(m, n)$. Table 2 reports the individual fraction of the turbulent kinetic energy and the cumulative energies of the velocity fluctuations of the first 10 most energetic modes of the decomposition ( $m$ and $n$ are the wavenumbers along $x$ and $z$, respectively and $q$ is the generic POD mode.

Table 2. Energy content of the first 10 eigenfunctions

\begin{tabular}{llll}
\hline Index & Mode $(m, n, q)$ & Energy fraction & Energy sum \\
\hline 1 & $(0,1,1)$ & 0.03220 & 0.03220 \\
2 & $(0,2,1)$ & 0.02173 & 0.05393 \\
3 & $(0,2,2)$ & 0.01535 & 0.06929 \\
4 & $(1,1,1)$ & 0.01508 & 0.08437 \\
5 & $(1,2,1)$ & 0.01454 & 0.09891 \\
6 & $(0,3,1)$ & 0.01197 & 0.11089 \\
7 & $(1,3,1)$ & 0.01196 & 0.12286 \\
8 & $(1,2,2)$ & 0.01160 & 0.13446 \\
9 & $(0,4,1)$ & 0.01053 & 0.14499 \\
10 & $(1,4,1)$ & 0.00972 & 0.15472 \\
\hline
\end{tabular}

About $6.9 \%$ of the energy resides in the first three streamwise-independent modes $(m=0)$. The first mode that exhibits a streamwise dependence is the fourth.

Figure 1 shows two surfaces of constant streamwise velocity (the light surface is positive, the dark surface is negative streamwise velocity) reconstructed from the first most energetic eigenfunction. The visualization shows two structures elongated in the streamwise direction. Flow representations (not reported) of the second and third eigenfunctions have shown streamwise-independent structures similar to those of Figure 1, with appropriate repetitions according to the values of $m$ and $n$. Figure 2 shows surfaces of constant streamwise velocity reconstructed from the fourth most energetic eigenfunction of the decomposition. This is the first streamwise-dependent eigenfunction. The visualization shows couples of bean-shaped quasi-streamwise flow structures, where one of the structure of each couple is lower with respect to the first (more displaced toward the center of the channel). Flow representations (not reported) of the fifth eigenfunction have shown bean-shaped structures aligned in the streamwise direction, similar to those of Figure 2.

Figure 3 shows the flow structure - identified in terms of surfaces of constant streamwise velocity - formed by the sum of the first five most energetic POD modes (three $x$-independent structures of the type shown in Figure 1 and two $x$-dependent turbulent structures of the type shown in Figure 2) at $t^{+}=72$. Two dominant structures elongated in the streamwise direction are visible. With particular reference 


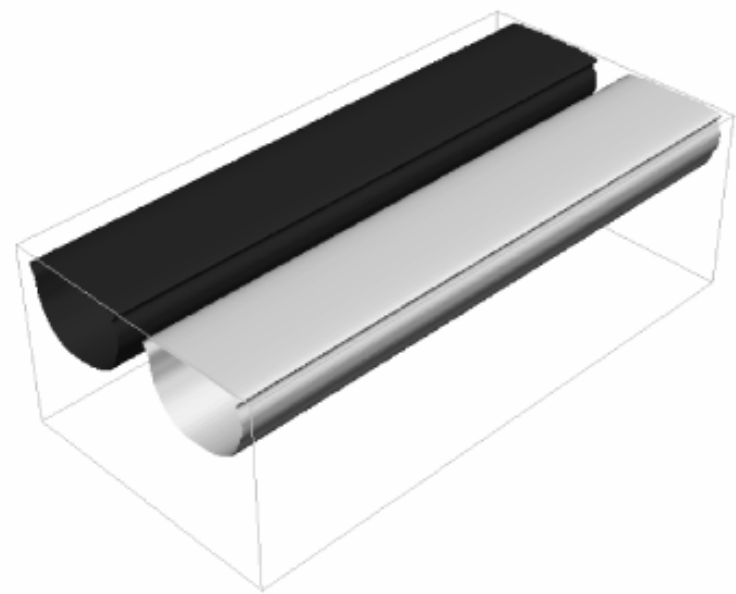

Fig. 1. Surfaces of constant $x$-velocity reconstructed from the first POD mode

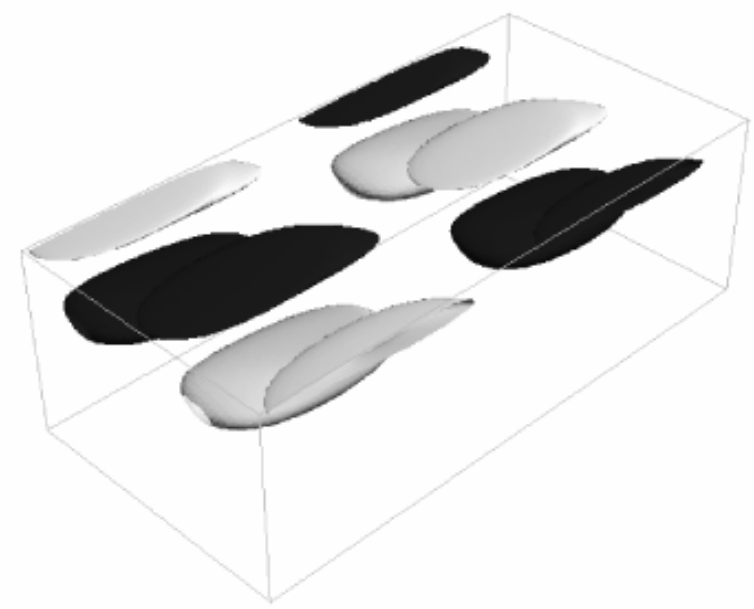

Fig. 2. Surfaces of constant $x$-velocity reconstructed from the fourth POD mode 


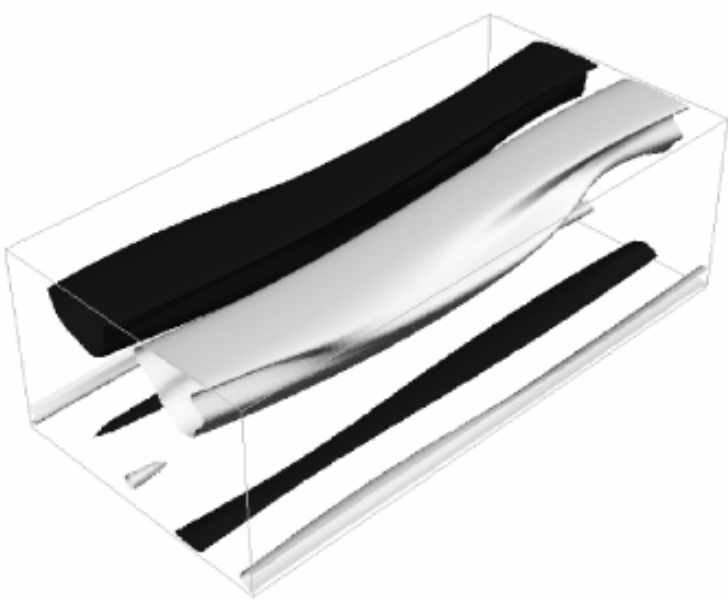

Fig. 3. Surfaces of constant $x$-velocity reconstructed from the first five POD modes at $t^{+}=72$

to the positive (light) surface it clearly appears that the shape of the basic streamwiseelongated structure is altered because of the interaction with the travelling beanshaped quasi-streamwise modes. This is the basic mechanism of evolution in time of the turbulent flow structures, that has been revealed due to the fact that the flow phenomena have been described in terms of dominant (most energetic) turbulent structures (the POD modes in this context).

\section{Concluding Remarks}

The analysis of the flow field of numerically simulated turbulent channel flow is performed in terms of flow modes determined with the POD technique. The dynamic properties of the POD modes are investigated, revealing the basic mechanism of evolution in time of the turbulent structures. This mechanism consists in the mutual interaction of two different types of modes, streamwise-independent structures and bean-shaped, quasi-streamwise modes aligned in the streamwise direction.

\section{References}

1. Kolmogorov A.N.: The local structure of turbulence in incompressible viscous fluid for very large Reynolds numbers. Dokl. Akad. Nauk. SSSR 30 (1941) 301

2. Pumir A. \& Shraiman B.I.: Persistent small scale anisotropy in homogeneous shear flows. Phys. Rev. Lett. 75 (1996) 3114 
3. Garg S. \& Warhaft Z.: On the small scale structure of simple shear flow. Phys. Fluids $\mathbf{1 0}$ (1998) 662

4. Shen X. \& Warhaft Z.: The anisotropy of the small scale structure in high Reynolds number $\left(R_{\lambda} \approx 1000\right.$ ) turbulent shear flow. Phys. Fluids 12 (2000) 2976

5. Podvin B. \& Lumley J.L.: A low-dimensional approach for the minimal flow unit. J. Fluid Mech., 362 (1998) 121

6. Omurtag A. \& Sirovich L.: On low-dimensional modeling of channel turbulence. Theor. Comp. Fluid Dyn. 13 (1999) 115

7. Alfonsi G., Passoni G., Pancaldo L. \& Zampaglione D.: A spectral-finite difference solution of the Navier-Stokes equations in three dimensions. Int. J. Num. Meth. Fluids $\mathbf{2 8}$ (1998) 129

8. Passoni G., Alfonsi G., Tula G. \& Cardu U.: A wavenumber parallel computational code for the numerical integration of the Navier-Stokes equations. Parall. Comp. 25 (1999) 593

9. Passoni G., Cremonesi P. \& Alfonsi G.: Analysis and implementation of a parallelization strategy on a Navier-Stokes solver for shear flow simulations. Parall. Comp. 27 (2001) 1665

10. Passoni G., Alfonsi G. \& Galbiati M.: Analysis of hybrid algorithms for the Navier-Stokes equations with respect to hydrodynamic stability theory. Int. J. Num. Meth. Fluids $\mathbf{3 8}$ (2002) 1069

11. Dean R.B.: Reynolds number dependence of skin friction and other bulk flow variables in two-dimensional rectangular duct flow. J. Fluids Eng. 100 (1978) 215

12. Berkooz G., Holmes P., Lumley J.L.: The Proper Orthogonal Decomposition in the analysis of turbulent flows. Ann. Rev. Fluid Mech. 25 (1993) 539

13. Sirovich L.: Turbulence and the dynamics of coherent structures. Part I: coherent structures. Part II: symmetries and transformations. Part III: dynamics and scaling. Quart. Appl. Math. 45 (1987) 561 